ИЗВЕСТИЯ АКАДЕМИИ НАУК ЭСТОНСКОИ ССР. ТОМ ХIV СЕРИЯ ФИЗИКО-МАТЕМАТИЧЕСКИХ И ТЕХНИЧЕСКИХ НАУК. 1965, N2 1

\title{
О НЕКОТОРЫХ ПРИБЛИЖЕННЫХ ФОРМУЛАХ, НАИЛУЧШИХ В СМЫСЛЕ МЕРЫ ВИНЕРА
}

\section{Сообщение I}

В классическом анализе существует целый ряд методов получения приближенных формул численного анализа. Например, для вычисления значения функции в данной точке можно использовать интерполяционный многочлен Лагранжа, многочлен наилучшего равномерного приближения, отрезок ряда Фурье и пр.; для вычисления определенного интеграла имеется множество различных по идее формул и т. д. Для каждой такой формулы существует и своя оценка ошибки, которая всегда основана на структурных свойствах функции (или класса функций). Использование этих оценок требует громоздкой вычислительной работы. В приложениях, когда функщии заданы таблично, почти всегда невозможно угадать порядок их дифференцируемости (или принадлежность к какому-то классу типа Липшица).

В некоторых случаях заранее известно, что функции являются плохими (недифференцируемыми), но зато известна некоторая вероятностная информация (например, их распределение). Поэтому возникает необходимость вывести приближенные формулы, которые используют лишь эту информацию. Функции рассматриваются как элементы пространства траекторий случайных процессов, т. е. пространства с вероятностной мерой. Так как ошибка формулы является функционалом на этом пространстве, то дисперсию этого функционала можно рассматривать как оценку ошибки.

В статьях [5-7] дан разбор некоторых известных методов для случая, когда функции являются траекториями стационарного случайного процесса.

В работах Сульдина $[2,3]$ исследовано приближение траекторий винеровского процесса и других сходных процессов. В статье ${ }^{[4]}$ даны некоторые обобщения на случай функций $n$ переменных.

В настоящей работе мы рассмотрим некоторые формулы приближения элементов винеровского пространства и других сходных пространств. Основное внимание мы уделяем формулам, которые используют значения функции лишь в некоторых точках, как наиболее важным при практических вычислениях. Надо сказать, что при использовании большого количества точек формулы (и оценки ошибки) очень усложняются.

Как будет видно из дальнейшего, выведенные нами формулы значительно сложнее известных приближенных формул, а разница в дисперсиях невелика. Нам кажется, что при приближении рассматриваемых ниже случайных величин всегда имеет смысл попытаться уменьшить шаг и воспользоваться простейшими формулами (линейной интерполяцией, формулой, трапеций и т. д.). Использование нижеследующих формул можно рекомендовать лишь в случае, когда уменьшение шага невозможно.

В первой части работы мы рассмотрим интерполяцию и вычисление определенного интеграла, а во второй части - приближение рядами типа Фурье, формулы дифференцирования и формулу Тэйлора.

Необходимые сведения о мере Вннера можно найти в статье [1]. 


\section{1. Введение}

Пусть C - пространство всех непрерывных на отрезке $[0,1]$ функции $x(t)$, удовлетворяющих условию $x(0)=0$, и пусть на пространстве $C$ определена мера Винера. Известно, что

$$
\begin{aligned}
& E x(t)=\int_{C} x(t) d_{W} x=0, \\
& R(s, t)=E x(s) \cdot x(t)=\int_{C} x(s) x(t) d_{W} x=\min (s, t), \\
& D x(t)=R(t, t)=t .
\end{aligned}
$$

Пусть оператор $T$ осуществляет однозначное отображение пространства $C$ на пространство $C^{T} \subset C$. Мера Винера индуцирует на пространстве $C^{T}$ новую меру $\mu(y)$, при этом

$$
\int_{C^{T}} F(y) d \mu(y)=\int_{C} F(T x) d_{\varpi x}
$$

В дальнейшем мы рассмотрим следующие пространства.

1. Пространство $C$. Отметим, что множество всех функций, дифференцируемых хотя бы в одной точке, образует в $C$ множество нулевой меры.

2. Пространство $C^{(m)}$ всех $m$ раз непрерывно дифференцируемых функций, удовлетворяющих условиям $y(0)=y^{\prime}(0)=\ldots=y^{(m)}(0)=0$. Оно порождается преобразованием

$$
T: x(t) \rightarrow y(t)=\frac{1}{(m-1) !} \int_{0}^{t}(t-u)^{m-1} x(u) d u .
$$

При этом

$$
\begin{aligned}
R(s, t)=\frac{1}{[(m-1) !]^{2}} & \int_{C} \int_{0}^{s}(s-u)^{m-1} x(u) d u \int_{0}^{t}(t-v)^{m-1} x(v) d v d \varpi x= \\
& =\frac{1}{(m !)^{2}} \int_{0}^{\min (s, t)}(s-u)^{m}(t-u)^{m} d u
\end{aligned}
$$

H

$$
E y^{(p)}(s) \cdot y^{(q)}(t)=\frac{1}{(m-p) !(m-q) !} \int_{0}^{\min (s, t)}(s-u)^{m-p}(t-u)^{m-q} d u .
$$


Для случая $m=1$ :

$$
R(s, t)=\left\{\begin{array}{l}
s t \min (s, t)-\frac{s}{2} \min ^{2}(s, t)-\frac{t}{2} \min ^{2}(s, t)+\frac{1}{3} \min ^{3}(s, t), \\
\frac{1}{2} s^{2} t-\frac{1}{6} s^{3}, \quad \text { если } s<t, \\
\frac{1}{3} s^{3}, \quad \text { если } s=t,
\end{array}\right.
$$

и

$$
E y^{\prime}(t) \cdot y(s)=s \min (s, t)-\frac{1}{2} \min ^{2}(s, t) .
$$

В случае $m=2$ :

$$
\begin{aligned}
& R(s, t)= \begin{cases}\frac{1}{4}\left[s^{2} t^{2} \min (s, t)-\left(s t^{2}+s^{2} t\right) \min ^{2}(s, t)+\frac{s^{2}+4 s t+t^{2}}{3} \times\right. \\
\left.\times \min ^{3}(s, t)-\frac{s+t}{2} \min ^{4}(s, t)+\frac{1}{5} \min ^{5}(s, t)\right] \\
\frac{s^{3} t^{2}}{12}-\frac{s^{4} t}{24}+\frac{s^{5}}{120}, & \text { если } s<t \\
\frac{1}{20} s^{5}, & \text { если } s=t\end{cases} \\
& E y^{\prime}(t) \cdot y(s)= \begin{cases}\frac{s^{3} t}{6}-\frac{s^{4}}{24}, & \text { если } s<t \\
\frac{s^{4}}{8}, & \text { если } s=t \\
\frac{s^{2} t^{2}}{4}-\frac{s t^{3}}{6}+\frac{t^{4}}{24}, & \text { если } t<s\end{cases} \\
& E y^{\prime \prime}(t) \cdot y(s)= \begin{cases}\frac{s^{3}}{6}, & \text { если } s \leqslant t, \\
\frac{s^{2} t}{2}-\frac{s t^{2}}{2}+\frac{t^{3}}{6}, & \text { если } t<s ;\end{cases} \\
& E y^{\prime}(t) \cdot y^{\prime}(s)= \begin{cases}\frac{s^{2} t}{2}-\frac{s^{3}}{6}, & \text { если } s<t, \\
\frac{s^{3}}{3}, & \text { если } s=t\end{cases} \\
& E y^{\prime \prime}(t) \cdot y^{\prime}(s)= \begin{cases}\frac{s^{2}}{2}, & \text { если } s \leqslant t, \\
s t-\frac{t^{2}}{2}, & \text { если } t<s .\end{cases}
\end{aligned}
$$

3. Пространство $C^{(+)}$, порожденное преобразованием

$$
T: y(t)=x^{2}(t) .
$$

Рассмотрим центрированную случайную функцию 


$$
\begin{gathered}
z(t)=y(t)-t: \\
\int_{C}\left[x^{2}(t)-t\right] d_{W} x=0 . \\
R(s, t)=\int_{C}\left[x^{2}(t)-t\right]\left[x^{2}(s)-s\right] d_{W} x=2 \min ^{2}(s, t) .
\end{gathered}
$$

4. Пространство $C_{1, x}$ с нормированной условной мерой Винера. Функции $y(t) \in C_{1, x}$ удовлетворяют условиям $y(0)=0, y(1)=X$ и порождаются преобразованием

Так как

$$
T: y(t)=x(t)-t x(1)+t X
$$

$$
\int_{C_{1, X}} y(t) d_{W(1, X)}^{*} x=t X,
$$

то рассмотрим опять функции

$$
z(t) \doteq y(t)-t X
$$

для которых

$$
R(s, t)=\min (s, t)-s t .
$$

5. Пространство $C_{1, X}^{(1)}$. Если $y(t) \in C_{1, X}^{(1)}$, то $y^{\prime}(t) \in C_{1, x}$.

$$
T: y(t)=\int_{0}^{t} x(u) d u-\frac{t^{2}}{2} x(1)+\frac{t^{2}}{2} X
$$

Если $z(t)=y(t)-\frac{t^{2}}{2} X$,

TO

$$
E z(t)=0
$$

и

$$
R(s, t)= \begin{cases}\frac{s^{2} t}{2}-\frac{s^{3}}{6}-\frac{s^{2} t^{2}}{4}, & \text { если } s<t \\ \frac{s^{3}}{3}-\frac{s^{4}}{4}, & \text { если } s=t\end{cases}
$$

Отметим еще, что

$$
E z^{\prime}(t) \cdot z(s)= \begin{cases}\frac{s^{2}}{2}-\frac{s^{2} t}{2}, & \text { если } s<t, \\ \frac{s^{2}}{2}-\frac{s^{3}}{2}, & \text { если } s=t, \\ s t-\frac{t^{2}}{2}-\frac{s^{2} t}{2}, & \text { если } t<s .\end{cases}
$$


Пусть $y$ - пространство с вероятностной мерой $\mu(y)$ и пусть $H-$ гильбертово пространство всех суммируемых с квадратом функционалов, определенные на $y$, т. е., если $F \in H$, то

$$
(F, F)=\int_{\boldsymbol{y}}|F(y)|^{2} d \mu(y)<\infty .
$$

Рассмотрим приближенную формулу

$$
F(y) \approx \sum_{i=1}^{n} \varphi_{i} V_{i}(y)
$$

где $V_{i}(y) \in H$ и $F(y) \in H$. Если в формуле (1.12) коэффициенты $\varphi_{i}$ определены так, что дисперсия ошибки будет минимальной

$$
\delta=\int_{y}\left[F(y)-\sum_{i=1}^{n} \varphi_{i} V_{i}(y)\right]^{2} d \mu(y)=\min ,
$$

то мы назовем формулу (1.12) наилучшей (в среднеквадратическом смысле). Коэффициенты $\varphi_{i}$ можно определить из системы

$$
\sum_{i=1}^{n} \varphi_{i}\left(V_{i}, V_{i}\right)=\left(F, V_{j}\right) \quad(j=1, \ldots, n)
$$

Если $V_{i}(y)$ ортогональны, то просто

$$
\varphi_{i}=\frac{1}{\left(V_{i}, V_{i}\right)}\left(F, V_{i}\right)=\frac{1}{D_{i}}\left(F, V_{i}\right) \text {. }
$$

Пусть $y$ - пространство траектории $y(t)$ случайного процесса на отрезке $[0,1]$ (в дальнейшем $У$ - одно из вышеуказанных пространств). Пусть $F(y)=y(t), V_{i}(y)=y\left(t_{i}\right) \quad\left(\right.$ где $\left.0=t_{0}<t_{1}<\ldots<t_{n} \leqslant 1\right)$ и

$$
R(s, t)=\int_{y} y(s) y(t) d \mu(y)
$$

Предположим, что

$$
\int_{\dot{y}} y(t) d \mu(y)=0
$$

Функционалы $y\left(t_{i}\right)$ не являются ортогональными, но их можно ортогонализировать. Нетрудно видеть, что все величины в формуле (1.12) можно определить рекуррентно следующим образом:

$$
\begin{aligned}
& V_{i}(y)=y\left(t_{i}\right)-\sum_{k=1}^{i-1} V_{k}(y) \varphi_{k}\left(t_{i}\right), \\
& D_{i}=R\left(t_{i}, t_{i}\right)-\sum_{k=1}^{i-1} D_{k} \varphi_{k}^{2}\left(t_{i}\right),
\end{aligned}
$$




$$
\varphi_{i}(t)=\frac{1}{D_{i}}\left[R\left(t, t_{i}\right)-\sum_{k=1}^{i-1} D_{k} \varphi_{k}(t) \varphi_{k}\left(t_{i}\right)\right]
$$

Здесь функционалы $V_{i}(y)$ ортогональны. При других выборах функционала $F(y)$, можно использовать ту же самую систему $V_{i}(y)$, но наилучшие коэффицненты определяются по формуле (1.13). Дисперсия ошибки:

$$
\delta=\int_{\boldsymbol{y}}\left[F(y)-\sum_{i=1}^{n} \varphi_{i} V_{i}(y)\right]^{2} d \mu(y)=(F, F)-\sum_{i=1}^{n} \frac{1}{D_{i}}\left(F, V_{i}\right)^{2}
$$

В дальнейшем ошибка приближенной формулы (1.12)

$$
\eta=F(y)-\sum_{n=1}^{n} \varphi_{i} V_{i}(y)
$$

часто является гауссовской случайной величиной с нулевым математическим ожиданием и дисперсией $\delta$.

Но тогда

$$
P(|\eta|<\varepsilon)=\frac{2}{\delta \sqrt{2 \pi}} \int_{0}^{\varepsilon} \exp \left(-\frac{t^{2}}{2 \delta^{2}}\right) d t=\frac{2}{\sqrt{2 \pi}} \int_{0}^{\frac{\varepsilon}{\delta}} \exp \left(-\frac{t^{2}}{2}\right) d t
$$

Если $\delta=\min$, то $P(|\eta|<\varepsilon)=\max$.

\section{2. Интерполирование функции}

Пусть $x(t) \in C$ и пусть $0=t_{0}<t_{1}<\ldots<t_{n} \leqslant 1$. Рассмотрим приближенную формулу вида

$$
x(t) \approx \sum_{i=1}^{n} C_{i} x\left(t_{i}\right)
$$

По формулам (1.14) - (1.16) легко найти, что

$$
\begin{aligned}
& V_{i}=x\left(t_{i}\right)-x\left(t_{i-1}\right) \\
& D_{i}=t_{i}-t_{i-1} \\
& \varphi_{i}(t)=\frac{1}{t_{i}-t_{i-1}}\left[\min \left(t, t_{i}\right)-\min \left(t, t_{i-1}\right)\right] .
\end{aligned}
$$

Поэтому наилучшей формулой интерполяции является

$$
x(t) \approx \sum_{i=1}^{n} \frac{1}{t_{i}-t_{i-1}}\left[\min \left(t, t_{i}\right)-\min \left(t, t_{i-1}\right)\right]\left[x\left(t_{i}\right)-x\left(t_{i-1}\right)\right] .
$$

Если $t_{i} \leqslant t \leqslant t_{i+1}$, то 


$$
x(t) \approx \frac{t_{i+1}-t}{t_{i+1}-t_{i}} x\left(t_{i}\right)+\frac{t-t_{i}}{t_{i+1}-t_{i}} x\left(t_{i+1}\right)
$$

Вероятностный смысл формулы (2.2) очевиден: если приращения функции имеют независимые гауссовские распределения, а значения функции на концах отрезка $\left[t_{i}, t_{i+1}\right]$ равны $x\left(t_{i}\right)$ и $x\left(t_{i+1}\right)$, то ее математическое ожидание как раз выражается формулой (2.2).

Дисперсия формулы (2.2):

$$
\delta(t)=\frac{\left(t_{i+1}-t\right)\left(t-t_{i}\right)}{t_{i+1}-t_{i}}
$$

при этом

$$
\max _{t_{i} \leqslant t \leqslant t_{i+1}} \delta(t)=\delta\left(\frac{t_{i}+t_{i+1}}{2}\right)=\frac{t_{i+1}-t_{i}}{4} .
$$

Для сравнения приводим дисперсию $\delta^{\prime}(t)$ на отрезке $t \in\left[\frac{1}{3}, \frac{2}{3}\right]$ при приближении интерполяционным многочленом третьей степени при узлах $t_{1}=0, t_{2}=\frac{1}{3}, t_{3}=\frac{2}{3}, t_{4}=1$ :

$$
\delta^{\prime}(t)=\frac{81}{2} t^{6}-\frac{243}{2} t^{5}+153 t^{4}-\frac{207}{2} t^{3}+\frac{73}{2} t^{2}-5 t,
$$

$\max \delta^{\prime}(t)=\delta^{\prime}\left(\frac{1}{2}\right)=\frac{11}{128}$.

$1 / 3 \leqslant t \leqslant 2 / 3$

Пусть $y(t) \in C^{(1)}$. Рассмотрим лишь случай двух точек $\left(t_{1} \leqslant t \leqslant t_{2}\right)$ :

$$
y(t) \approx C_{1} y\left(t_{1}\right)+C_{2} y\left(t_{2}\right)
$$

По формулам (1.1) и (1.14) - (1.16) найдем:

$$
\begin{aligned}
& V_{1}=y\left(t_{1}\right), D_{1}=\frac{t_{1}^{3}}{3}, \quad \varphi_{1}(t)=\frac{1}{D_{1}}\left(\frac{1}{2} t_{1}^{2} t-\frac{1}{6} t_{1}^{3}\right) \\
& V_{2}=y\left(t_{2}\right)-y\left(t_{1}\right) \frac{1}{t_{1}}\left(\frac{3}{2} t_{2}-\frac{1}{2} t_{1}\right) \\
& D_{2}=\frac{t_{2}^{3}}{3}-\frac{t_{1}}{3}\left(\frac{3}{2} t_{2}-\frac{1}{2} t_{1}\right)^{2}, \\
& \varphi_{2}(t)=\frac{1}{D_{2}}\left[t^{2}\left(\frac{1}{2} t_{2}-\frac{1}{6} t\right)-t_{1}\left(\frac{3}{2} t-\frac{1}{2} t_{1}\right)\left(\frac{1}{2} t_{2}-\frac{1}{6} t_{1}\right)\right] .
\end{aligned}
$$

Наилучшая формула по точкам $t_{1}$ и $t_{2}$ :

$$
y(t) \approx V_{1} \varphi_{1}(t)+V_{2} \varphi_{2}(t) .
$$

Если $t_{1}=\frac{k_{-}-1}{n}, t_{2}=\frac{k}{n}$ и $t=\frac{2 k-1}{2 n}$, то

$$
y\left(\frac{2 k-1}{2 n}\right) \approx \frac{12 k^{2}-2 k-1}{8(3 k+1)(k-1)} y\left(\frac{k-1}{n}\right)+\frac{6 k-1}{4(3 k+1)} y\left(\frac{k}{n}\right)
$$


с дисперсией $\delta=\frac{1}{48 n^{3}}-\frac{3}{64 n^{3}(3 k+1)}$. При линейной интерполяции соответствующая дисперсия* $\delta^{\prime}=\frac{1}{48 n^{3}}$.

Сделаем одно общее замечание о пространстве $C^{(1)}$. Рассмотрим систему функционалов

$$
U_{1}=\frac{y_{1}}{t_{1}}, \quad U_{k}=\frac{y_{k}-y_{k-1}}{t_{k}-t_{k-1}}-\frac{y_{k-1}-y_{k-2}}{t_{k-1}-t_{k-2}},
$$

где $y_{k}=y\left(t_{k}\right)$.

Известно [3], что

$$
\begin{array}{cl}
\left(U_{k}, U_{k}\right)=\frac{t_{k}-t_{k-2}}{3} ; & \left(U_{k}, U_{k-1}\right)=\frac{t_{k-1}-t_{k-2}}{6} ; \\
\left(U_{k}, U_{l}\right)=0 \quad(l \Longleftarrow k-1, k, k+1) .
\end{array}
$$

Исходя из системы $\left\{U_{k}\right\}$, можно рекуррентно найти ортогональную систему $\left\{V_{k}\right\}$ :

где

$$
V_{1}=U_{1}, V_{k}=U_{k}+\alpha_{k-1} V_{k-1},
$$

$$
\alpha_{k-1}=-\frac{\left(U_{k}, U_{k-1}\right)}{\left(U_{k-1}, V_{k-1}\right)}=-\frac{\left(U_{k}, U_{k-1}^{\prime}\right)}{\left(U_{k-1}, U_{k-1}\right)+\alpha_{k-2}\left(U_{k-1}, U_{k-2}\right)}
$$

Если $t_{k}=\frac{k}{n}$, то $\alpha_{1}=-\frac{1}{2}, \alpha_{k}=-\frac{1}{4+\alpha_{k-1}}$.

При системе $\left\{V_{k}\right\}$ :

$$
\begin{gathered}
D_{k}=\left(U_{k}, U_{k}\right)+2 \alpha_{k-1}\left(U_{k}, U_{k-1}\right)+\alpha_{k-1}^{2}\left(V_{k-1}, V_{k-1}\right) \\
\text { (если } \left.t_{k}=\frac{k}{n}, \text { то } D_{k}=\frac{2}{3} n+\alpha_{k-1} \frac{1}{3 n}+\alpha_{k-1}^{2} D_{k-1}\right), \\
\varphi_{k}(t)=\frac{1}{D_{k}}\left\{\alpha_{k-1} \varphi_{k-1}(t) D_{k-1}+\frac{1}{t_{k}-t_{k-1}}\left[R\left(t, t_{k}\right)-R\left(t, t_{k-1}\right)\right]-\right. \\
\left.-\frac{1}{t_{k-1}-t_{k-2}}\left[R\left(t, t_{k-1}\right)-R\left(t, t_{k-2}\right)\right]\right\} .
\end{gathered}
$$

Если $y(t) \in C^{(2)}$, то

$$
\begin{aligned}
& V_{1}=y\left(t_{1}\right), \quad D_{1}=\frac{t_{1}^{5}}{20}, \quad \varphi_{1}(t)=\frac{1}{D_{1}} \frac{t_{1}^{3}}{12}\left(t^{2}-\frac{1}{2} t_{1} t+\frac{1}{10} t_{1}^{2}\right), \\
& V_{2}=y\left(t_{2}\right)-y\left(t_{1}\right) \frac{5\left(t_{2}^{2}-\frac{1}{2} t_{1} t_{2}+\frac{1}{10} t_{1}^{2}\right)}{3 t_{1}^{2}},
\end{aligned}
$$

Большинство дисперсий обычных формул (обозначаемые через $\delta^{\prime}$ ) приводится в работе Сульдина [ $\left.{ }^{3}\right]$. 


$$
\begin{aligned}
& D_{2}=\frac{1}{20} t_{2}^{5}-\frac{5 t_{1}}{36}\left(t_{2}^{2}-\frac{1}{2} t_{1} t_{2}+\frac{1}{10} t_{1}^{2}\right)^{2} \\
& \varphi_{2}(t)=\frac{1}{D_{2}} \frac{1}{12}\left[t^{3} t_{2}^{2}-\frac{1}{2} t^{4} t_{2}+\frac{1}{10} t^{5}-\frac{5}{3} t_{1}\left(t^{2}-\frac{1}{2} t_{1} t+\frac{1}{10} t_{1}^{2}\right) \times\right. \\
& \left.\times\left(t_{2}^{2}-\frac{1}{2} t_{1} t_{2}+\frac{1}{10} t_{1}^{2}\right)\right] .
\end{aligned}
$$

Если $t_{1}=\frac{k}{n}, t_{2}=\frac{k+1}{n}$ и $t=\frac{2 k+1}{2 n}$, то

$$
\begin{aligned}
& V_{1}=y\left(\frac{k}{n}\right), D_{1}=\frac{1}{20} \frac{k^{5}}{n^{5}}, \varphi_{1}=\frac{1}{D_{1}} \frac{k^{3}\left(12 k^{2}+15 k+5\right)}{240 n^{5}}, \\
& V_{2}=y\left(\frac{k+1}{n}\right)-y\left(\frac{k}{n}\right) \frac{6 k^{2}+15 k+10}{6 k^{2}}, \\
& D_{2}=\frac{15 k^{3}+60 k^{2}+80 k+36}{720 n^{5}}, \\
& \varphi_{2}=\frac{1}{D_{2}} \frac{120 k^{3}+360 k^{2}+320 k+93}{16 \cdot 720 n^{5}} .
\end{aligned}
$$

В этом случае

$$
\begin{gathered}
y\left(\frac{2 k+1}{2 n}\right) \approx \\
+\frac{720 k^{5}+3600 k^{4}+6960 k^{3}+6498 k^{2}+2925 k+510}{96 k^{2}\left(15 k^{3}+60 k^{2}+80 k+36\right)} y\left(\frac{k}{n}\right)+ \\
+\frac{120 k^{3}+360 k^{2}+320 k+93}{16\left(15 k^{3}+60 k^{2}+80 k+36\right)} y\left(\frac{k+1}{n}\right),
\end{gathered}
$$

a

$$
\delta=\frac{1600 k^{4}+5520 k^{3}+6480 k^{2}+3200 k+573}{61440 n^{5}\left(15 k^{3}+60 k^{2}+80 k+36\right)} .
$$

Линейная интерполяция дает дисперсию $\delta^{\prime}=\frac{60 k+203}{3840 n^{5}}$.

Рассмотрим теперь случайную функцию $z(t)=y(t)-t$ с корреляционной функцией $R(s, t)=2 \min ^{2}(s, t)$ (т. е. $\left.x^{2}(t)=y(t) \in C^{(+)}\right)$. Методом индукции легко найдем:

$$
\begin{aligned}
& V_{i}=x^{2}\left(t_{i}\right)-x^{2}\left(t_{i-1}\right)-\left(t_{i}-t_{i-1}\right), \\
& D_{i}=2\left(t_{i}^{2}-t_{i-1}^{2}\right), \\
& \varphi_{i}(t)=\frac{1}{t_{i}^{2}-t_{i-1}^{2}}\left[\min ^{2}\left(t, t_{i}\right)-\min ^{2}\left(t, t_{i-1}\right)\right],
\end{aligned}
$$

поэтому

$$
\begin{aligned}
x^{2}(t) \approx t+ & \sum_{i=1}^{n}\left[x^{2}\left(t_{i}\right)-x^{2}\left(t_{i-1}\right)-\left(t_{i}-t_{i-1}\right)\right] \frac{1}{t_{i}^{2}-t_{i-1}^{2}} \times \\
& \times\left[\min ^{2}\left(t, t_{i}\right)-\min ^{2}\left(t, t_{i-1}\right)\right] .
\end{aligned}
$$


Если $t_{i} \leqslant t \leqslant t_{i+1}$, то

$$
x^{2}(t) \approx \frac{t_{i+1}^{2}-t^{2}}{t_{i+1}^{2}-t_{i}^{2}} x^{2}\left(t_{i}\right)+\frac{t^{2}-t_{i}^{2}}{t_{i+1}^{2}-t_{i}^{2}} x^{2}\left(t_{i+1}\right)+\frac{\left(t-t_{i}\right)\left(t_{i+1}-t\right)}{t_{i}+t_{i+1}}
$$

Дисперсия полученной формулы

$$
\begin{gathered}
\delta(t)=\frac{2\left(t_{i+1}^{2}-t^{2}\right)\left(t^{2}-t_{i}^{2}\right)}{t_{i+1}^{2}-t_{i}^{2}} \\
\max \delta(t)=\delta\left(\sqrt{\frac{t_{i}^{2}+t_{i+1}^{2}}{2}}\right)=\frac{t_{i+1}^{2}-t_{i}^{2}}{2} .
\end{gathered}
$$

При использовании формулы линейной интерполячции

$$
x^{2}(t) \approx \frac{t_{i+1}-t}{t_{i+1}-t_{i}} x^{2}\left(t_{i}\right)+\frac{t-t_{i}}{t_{i+1}-t_{i}} x^{2}\left(t_{i+1}\right)
$$

дисперсия $\delta^{\prime}(t)=\frac{4 t\left(t_{i+1}-t\right)\left(t-t_{i}\right)}{t_{i+1}-t_{i}}$.

$$
\delta(t)-\delta^{\prime}(t)=-\frac{2}{t_{i+1}^{2}-t_{i}^{2}}\left(t-t_{i}\right)^{2}\left(t_{i+1}-t\right)^{2}<0 .
$$

Пусть $z(t)=y(t)-t X$, где $y(t) \in C_{1, X}$.

Методом индукции найдем:

$$
\begin{gathered}
V_{k}=y\left(t_{k}\right)-t_{k} X-\frac{1-t_{k}}{1-t_{k-1}}\left[y\left(t_{k-1}\right)-t_{k-1} X\right] \\
D_{k}=\frac{\left(1-t_{k}\right)\left(t_{k}-t_{k-1}\right)}{1-t_{k-1}}, \\
\varphi_{k}(t)=\frac{1}{D_{k}}\left[\min \left(t, t_{k}\right)-t t_{k}-\frac{1-t_{k}}{1-t_{k-1}}\left(\min \left(t, t_{k-1}\right)-t t_{k-1}\right)\right] .
\end{gathered}
$$

Если $t_{k} \leqslant t \leqslant t_{k+1}$, то

$$
y(t) \approx \frac{t_{k+1}-t}{t_{k+1}-t_{k}} y\left(t_{k}\right)+\frac{t-t_{k}}{t_{k+1}-t_{k}} y\left(t_{k+1}\right)
$$

с дисперсней (2.3).

При $t_{n} \leqslant t \leqslant 1$

$$
y(t) \approx \frac{1-t}{1-t_{n}} y\left(t_{n}\right)+\frac{t-t_{n}}{1-t_{n}} X
$$

Основываясь на вероятностных соображениях, эти результаты можно было заранее предвидеть. 
74

T. Тобиас

Пусть, наконец, $z(t)=y(t)-\frac{t 2}{2} X$, где $y(t) \in C_{1, X}^{(1)}$.

Здесь

$$
\begin{gathered}
V_{1}=z\left(t_{1}\right), \quad D_{1}=\frac{t_{1}^{3}}{12}\left(4-3 t_{1}\right), \quad \varphi_{1}(t)=\frac{1}{D_{1}} \frac{t_{1}^{2}}{2}\left(t-\frac{1}{3} t_{1}-\frac{t^{2}}{2}\right), \ldots \\
V_{2}=z\left(t_{2}\right)-z\left(t_{1}\right) \frac{6 t_{2}-2 t_{1}-3 t_{2}^{2}}{t_{1}\left(4-3 t_{1}\right)}, \\
D_{2}=\frac{t_{2}^{3}\left(4-3 t_{2}\right)}{12}-\frac{t_{1}}{12\left(4-3 t_{1}\right)}\left(6 t_{2}-2 t_{1}-3 t_{2}^{2}\right)^{2}, \\
\varphi_{2}(t)=\frac{1}{D_{2}}\left[\frac{1}{2} t^{2}\left(t_{2}-\frac{1}{3} t-\frac{t_{2}^{2}}{2}\right)-\frac{t_{1}}{2\left(4-3 t_{1}\right)}\left(t-\frac{1}{3} t_{1}-\frac{t^{2}}{2}\right)\left(6 t_{2}-2 t_{1}-3 t_{2}^{2}\right)\right] .
\end{gathered}
$$

Если $t_{1}=\frac{k-1}{n}, \quad t_{2}=\frac{k}{n}, \quad t=\frac{2 k-1}{2 n}$, то

$$
\begin{aligned}
& V_{1}=z\left(\frac{k-1}{n}\right), \quad D_{1}=\frac{(k-1)^{3}(4 n-3 k+3)}{12 n^{4}} \\
& \varphi_{1}=\frac{1}{D_{1}} \frac{(k-1)^{2}\left(16 k n-4 n-12 k^{2}+12 k-3\right)}{48 n^{4}}, \\
& V_{2}=z\left(\frac{k}{n}\right)-z\left(\frac{k-1}{n}\right) \frac{2 n+4 k n-3 k^{2}}{(k-1)}, \quad D_{2}=\frac{n+3 k n-3 k^{2}}{3 n^{3}(4 n-3 k+3)} \\
& \begin{aligned}
\varphi_{2}= & \frac{1}{D_{2}} \frac{24 k n+1}{48 n^{3}}\left(\frac{15 n-24 k^{2}-4 n-3}{4 n+3)},\right. \\
z\left(\frac{2 k-1}{2 n}\right) & \approx \frac{24 k^{2} n-4 k n+9 k^{2}-24 k^{3}-2 n}{16(k-1)\left(3 k n-3 k^{2}+n\right)} z\left(\frac{k-1}{n}\right)+ \\
& \quad+\frac{24 k n+15 k-24 k^{2}-4 n-3}{16\left(3 k n-3 k^{2}+n\right)} z\left(\frac{k}{n}\right)
\end{aligned}
\end{aligned}
$$

$$
\delta=\frac{192 k n^{2}-336 k^{2} n-80 n^{2}-72 n+312 k n+144 k^{3}-225 k^{2}+90 k-9}{768 n^{3}(4 n-3 k+3)\left(3 k n+n-3 k^{2}\right)} .
$$

Соответствующая дисперсия линейной интерполяции:

$$
\delta^{\prime}=\frac{1}{48 n^{3}}-\frac{1}{64 n^{4}} .
$$

Пример. $z\left(\frac{1}{2}\right) \approx \frac{443}{736} z\left(\frac{2}{5}\right)+\frac{332}{736} z\left(\frac{3}{5}\right)$,

$$
\delta=\frac{1}{120000} \frac{665}{46} ; \quad \delta^{\prime}=\frac{1}{120000} \frac{782}{46} \text {. }
$$




\section{3. Интегрирование функции}

Пусть мы имеем наилучшую формулу интерполяции предыдущего параграфа

$$
y(t) \approx \sum_{k=1}^{n} \varphi_{k}(t) V_{k}(y)
$$

где $V_{k}(y)$ ортогональны, а

$$
\varphi_{k}(t)=\frac{1}{D_{k}} \int_{C} T x(t) V_{k}(T x) d_{\mathbb{w}} x
$$

Рассмотрим нанлучшую формулу для приближения интеграла

$$
\int_{0}^{1} y(t) d t \approx \sum_{k=1}^{n} C_{k} V_{k}(y)
$$

тде

$$
C_{k}=\frac{1}{D_{k}} \int_{\dot{C}} \int_{0}^{1} T x(t) d t \cdot V_{k}(T x) d_{W} x
$$

В силу теоремы Фубини

$$
C_{k}=\frac{1}{D_{k}} \int_{0}^{1} \int_{C} T x(t) V_{k}(T x) d W x d t=\int_{0}^{1} \varphi_{k}(t) d t .
$$

Итак, наилучшую формулу для приближения интеграла мы получим лутем интегрирования наилучшей формулы интерполяции.

Интегрируя формулу (2.1), получим

$$
\int_{0}^{1} x(t) d t \approx \sum_{k=1}^{n-1} \frac{t_{k+1}-t_{k-1}}{2} x\left(t_{k}\right)+\left(1-\frac{t_{n}+t_{n-1}}{2}\right) x\left(t_{n}\right)
$$

с дисперсией

$$
\delta=\frac{1}{3}-\sum_{k=1}^{n}\left(1-\frac{t_{k}+t_{k-1}}{2}\right)^{2}\left(t_{k}-t_{k-1}\right) .
$$

Можно поставить задачу: найти узлы $t_{k}$, которые минимизируют дисперсию (3.1). Решением задачи является (см. [ $\left.\left.{ }^{2}\right]\right) t_{k}=\frac{2 k}{2 n+1}$. Мы получили следующую формулу наилучшего интегрирования в пространстве $C$ :

$$
\int_{0}^{1} x(t) d t \approx \frac{2}{2 n+1} \sum_{k=1}^{n} x\left(\frac{2 k}{2 n+1}\right)
$$

с дисперсией $\delta=\frac{1}{3(2 n+1)^{2}}$. 
Рассмотрим вычисление $\int_{0}^{1} x^{2}(t) d t$.

Так как

$$
\begin{gathered}
\int_{0}^{1}\left[\min ^{2}\left(t, t_{k}\right)-\min ^{2}\left(t, t_{\mathrm{k}-1}\right)\right] d t=\frac{t_{k}^{3}-t_{k-1}^{3}}{3}- \\
-t_{k-1}^{2}\left(t_{k}-t_{k-1}\right)+\left(t_{k}^{2}-t_{k-1}^{2}\right)\left(1-t_{k}\right),
\end{gathered}
$$

то из формулы (2.6) получим

$$
\int_{0}^{1} x^{2}(t) d t \approx \frac{1}{2}+\sum_{k=1}^{n} C_{k} \frac{\left[x^{2}\left(t_{k}\right)-x^{2}\left(t_{k-1}\right)-\left(t_{k}-t_{k-1}\right)\right]}{\sqrt{2\left(t_{k}^{2}-t_{k-1}^{2}\right)}},
$$

где

$$
C_{k}=\frac{2}{\sqrt{2\left(t_{k}^{2}-t_{k-1}^{2}\right)}}\left[\frac{t_{k}^{3}-t_{k-1}^{3}}{3}-t_{k-1}^{2}\left(t_{k}-t_{k-1}\right)+\left(t_{k}^{2}-t_{k-1}^{2}\right)\left(1-t_{k}\right)\right] .
$$

В данном случае $\delta=\frac{1}{3}-\sum_{k=1}^{n} C_{k}^{2}$.

При $t_{k}=\frac{k}{n}$ получим формулу Сульдина [3]:

$$
\begin{gathered}
\int_{0}^{1} x^{2}(t) d t \approx \frac{1}{n} \sum_{k=1}^{n} x^{2}\left(\frac{k}{n}\right)-\frac{1}{3 n} \sum_{k=1}^{n} \frac{1}{4 k^{2}-1} x^{2}\left(\frac{k}{n}\right)-\frac{3 n+2}{3 n(2 n+1)} x^{2}(1)+ \\
+\frac{1}{3 n^{2}} \sum_{k=1}^{n} \frac{k}{4 k^{2}-1}+\frac{1}{6 n(2 n+1)} .
\end{gathered}
$$

Пусть теперь $y(t) \in C_{1, x}$ и рассмотрим вычисление $\int_{0}^{1}[y(t)-t X] d t$.

Легко найти, что

$\int_{0}^{1}\left[\min \left(t, t_{k}\right)-t t_{k}-\left(\min \left(t, t_{k-1}\right)-t t_{k-1}\right) \frac{1-t_{k}}{1-t_{k-1}}\right] d t=\frac{\left(t_{k}-t_{k-1}\right)\left(1-t_{k}\right)}{2}$.

\section{Поэтому}

$$
\begin{aligned}
& \int_{0}^{1}[y(t)-t X] d t \approx \sum_{k=1}^{n}\left[y\left(t_{k}\right)-t_{k} X-\frac{1-t_{k}}{1-t_{k-1}}\left(y\left(t_{k-1}\right)-t_{k-1} X\right)\right] X \\
& \times \sqrt{\frac{1-t_{k-1}}{\left(1-t_{k}\right)\left(t_{k}-t_{k-1}\right)}} C_{k}
\end{aligned}
$$


где

$$
C_{k}=\sqrt{\frac{1-t_{k-1}}{\left(1-t_{k}\right)\left(t_{k}-t_{k-1}\right)}} \frac{\left(1-t_{k}\right)\left(t_{k}-t_{k-1}\right)}{2}
$$

или

$$
\int_{0}^{1} y(t) d t \approx \sum_{k=1}^{n-1} \frac{t_{k+1}-t_{k-1}}{2} y\left(t_{k}\right)+\frac{1-t_{n-1}}{2} y\left(t_{n}\right)+\frac{1-t_{n}}{2} X .
$$

Дисперсия формулы (3.2)

$$
\delta=\frac{1}{12}-\sum_{k=1}^{n} C_{k}^{2}=\frac{1}{12}-\sum_{k=1}^{n} \frac{\left(1-t_{k}\right)\left(t_{k}-t_{k-1}\right)\left(1-t_{k-1}\right)}{4} .
$$

Для определения узлов $t_{k}$, минимизирующих дисперсию (3.3), полуним систему

$$
\begin{gathered}
\frac{\partial \delta}{\partial t_{k}}=\left(1-t_{k-1}\right)\left(1-t_{k}\right)-\left(1-t_{k-1}\right)\left(t_{k}-t_{k-1}\right)-\left(t_{k+1}-t_{k}\right)\left(1-t_{k+1}\right)- \\
-\left(1-t_{k}\right)\left(1-t_{k+1}\right)=0, \quad(k=1, \ldots, n-1) \\
\frac{\partial \delta}{\partial t_{n}}=\left(1-t_{n-1}\right)\left(1-t_{n}\right)-\left(1-t_{n-1}\right)\left(t_{n}-t_{n-1}\right)=0,
\end{gathered}
$$

решением которой являются $t_{k}=\frac{k}{n+1} \quad(k=1, \ldots, n)$.

Ктак:

$$
\int_{0}^{1} y(t) d t \approx \frac{1}{n+1} \sum_{k=1}^{n} y\left(\frac{k}{n+1}\right)+\frac{1}{2(n+1)} X
$$

с дисперсией

$$
\delta=\frac{1}{12(n+1)^{2}}
$$

Для других пространств мы получили интерполяционные формулы, использующие значения функции лишь в двух точках, поэтому их интегрирование не может дать оптимального ответа для нашей проблемы. Но можно надеяться, что интегрирование формул предыдущего параграфа на соответствующих отрезках даст лучшие результаты, чем иснользование других известных простейших формул (формулы прямоугольников или трапеций).

Рассмотрим, например, пространство $C^{(1)}$. Пусть $t_{1}=\frac{k-1}{n}$ и $t_{2}=\frac{k}{n}$. Интегрируя (2.4) на отрезке $\left[\frac{k-1}{n}, \frac{k}{n}\right](k=1, \ldots, n)$ и прнводя подобные члены, получим

$$
\int_{0}^{1} y(t) d t \approx \frac{1}{n} \sum_{k=1}^{n} C_{k} y\left(\frac{k}{n}\right)
$$


где

$$
\begin{aligned}
& C_{k}=\frac{36 k^{3}+60 k^{2}+19 k+3}{4 k(3 k+1)(3 k+4)} \quad(k=1, \ldots, n-1), \\
& C_{n}=\frac{3 n}{2(3 n+1)} .
\end{aligned}
$$

Л И ТЕ Р А Т Р Р А

1. Гель фанд И. М., Я глом А. М., Успехи матем. наук, 11 , вып. $1(67), 77-114$ (1956).

2. С ульдин А. В., Изв. вузов, № 6 (13), 145-156 (1959); Изв. вузов, № 5 (18), $167-179$ (1960).

3. С ульдин А. В., Сб. Вероятностные методы и кибернетика, II, Изд. Казанск. ун-та, $1963,3-35$.

4. Т о б и а с Т., Изв. АН Эст. ССР. Сер. фнз.-мат. и техн. наук, № 1, 70-82 (1964).

5. B 1 a n c Ch., Comment. Math. Helv., No. 26, 225-241 (1952).

6. B I a n c Ch., Arch. Math., Nr. 5, 301-308 (1954).

7. Blanc Ch., Liniger W., Z. angew. Math. und Mech., 35, H. 4, 121-130 (1955).

Ннститут кибернетики

Академии наук Эстонской ССР
Поступила в редакцию 26/VI 1964

\section{T. TOBIAS}

\section{MOONINGAIST WIENERI MŌODU MOTTTES PARIMATEST LIGIKAUDSETEST VALEMITEST. I}

Artiklis vaadeldakse interpolatsioonivalemeid ja määratud integraali arvutamist Wieneri ruumis ning teistes mõōtudega ruumides. Valemi optimaalsuse kriteeriumiks võetakse dispersiooni minimaalsus.

\section{T. TOBIAS}

\section{SOME APPROXIMATION FORMULAE WHICH ARE THE BEST FROM THE STANDPOINT OF WIENER'S MFASURE. I}

In this paper we consider interpolation formulae and the approximation of thedefinite integral on Wiener's and other similar spaces. As a measure for the goodness of the formulae we use the mean square deviation. 